\title{
Biomarkers in Orthodontics: An Overview
}

\author{
Poonam Agrawal $^{1}$, Dhirja Goel ${ }^{2}$ \\ ${ }^{1}$ Professor, Dept. of Orthodontics and Dentofacial Orthopaedics, ${ }^{2}$ Reader, Dept. of Pediatric and Preventive \\ Dentistry, School of Dental Sciences, Sharda University, Greater Noida (UP)-201310
}

\begin{abstract}
A biomarker is a substance that is measured and evaluated objectively as an indicator of normal biologic processes, pathogenic processes, or pharmacologic responses to a therapeutic intervention. Tooth movement by orthodontic treatment is characterized by remodelling changes in the periodontal ligament, alveolar bone and gingiva. A reflection of these phenomenons can be found in the gingival crevicular fluid (GCF) of moving teeth, with significant elevations in the concentrations of its components like, cytokines, neurotransmitters, growth factors and arachidonic acid metabolites. Knowledge of biomarkers present in the GCF may be of clinical use leading to proper choice of mechanical stress for betterorthodontic treatment and lesser side effects.
\end{abstract}

Keywords: Biomarkers, orthodontic tooth movement, gingival crevicular fluid.

\section{Introduction}

Orthodontic treatment aims at the correction of dental irregularities and disharmony in jaw relations. Tooth movement induced by orthodontic force application is characterized by remodelling in the dental and periodontal tissues. ${ }^{1}$ Orthodontic tooth movement (OTM) is characterized by abrupt creation of compression and tension in periodontal ligament (PDL) (Goutoudi, Diza et al. 2004). These force-induced strains alter the PDL vascularity and blood flow, resulting in local synthesis and release of various key molecules, such as neurotransmitters, cytokines, growth factors, colonystimulating factors and arachidonic acid metabolites. These molecules can evoke many cellular responses by various cell types in and around teeth, providing a favourable micro-environment for tissue deposition or resorption (Simonet, Lacey et al. 1997; Cetin, Buduneli et al 2004). Gingival crevicular fluid (GCF) contains inflammatory products, bacterial products and products of tissue break down. Noninvasive procedures to determine the changes in salivary constituents are used to diagnose several diseases in clinical medicine. Thus, examination of GCF is an ideal method of evaluating the tissue destruction during orthodontic treatment. GCF arises at the gingival margin and can be described as a transudate or an exudate. Clinically GCF can be easily collected using platinum loops, filter paper strips, gingival washings and micropipettes. A number of GCF biomarkers are involved in bone remodeling during OTM. The data suggest that knowledge of the biomarkers present in the GCF may be of clinical use leading to proper choice of mechanical stress to improve and to shorten treatment time and avoid side effects.

Gingival crevicular fluid: Gingival crevicular fluid (GCF) is an exudate that can be harvested from the gingival sulcus, which offers a great potential as a source of factors associated with changes sand destruction in the underlying periodontium due to orthodontic force application. The early phase of orthodontic tooth movement involves an acute inflammatory response, characterized by periodontal vasodilation and migration of leukocytes out of periodontal ligament capillaries. The mechanism of bone resorption might also be related to the release of inflammatory mediators that can be detected in gingival crevicular fluid.

Biomechanism of orthodontic tooth movement: Two interrelated processes involved in OTM are bone bending and remodelling of the periodontal tissues, including the dental pulp, periodontal ligament, alveolar bone and gingiva. The applied force causes the compression of the alveolar bone and the PDL on one side (pressure), while on the opposite side the PDL is stretched (tension). ${ }^{[2]}$ Orthodontic forces change 
periodontal tissue vascularity leading to the synthesis of various signalling molecules and metabolites. The released molecules generate cellular responses around the teeth, providing a favourable microbiological environment for tissue deposition or resorption.

The pressure-tension theory proposed by Schwartz in 1932 is the simplest theory describing tooth movement on mechanical loading. On the pressure side, the biological events are as follows: disturbance of blood flow in the compressed PDL, cell death in the compressed area of the PDL (hyalinization), resorption of the hyalinized tissue by macrophages and undermining bone resorption by osteoclasts beside the hyalinized tissue, which ultimately results in tooth movement. ${ }^{2} \mathrm{On}$ the tension side, blood flow is activated where the PDL is stretched, which promote osteoblastic activity and osteoid deposition, which later mineralizes. The fluid flow hypothesis, describing a mechanism by which osteocytes respond to mechanical forces, states that locally evoked strain derived from the displacement of fluid in the canaliculi is very important. When loading occurs, interstitial fluid is squeezed through the thin layer of the non-mineralized matrix surrounding the cell bodies and cell processes, resulting in local strain at the cell membrane and activation of the affected osteocytes.

The sequence of events following orthodontic tooth movement can be characterized using suitable biomarkers. Proinflammatory cytokines: Interleukin-1 (IL-1), Interleukin- 6 (IL-6), Interleukin-8 (IL-8), tumornecrosisfactor- $\alpha$ (TNF- $\alpha$ ) and prostaglandin E (PGE). The analysis of the association between alkaline phosphatase (ALP) and bone metabolism, under healthy gingival conditions, is a suggestive indicator of histological and biochemical changes in bone turnover and therefore of the amount of tooth movement. Finally, specific properties of GCF ALP activity render this enzyme an interesting diagnostic tool in orthodontics.

Biomarkers of orthodontic tooth movement: A biomarker is a substance that is measured and evaluated objectively as an indicator of normal biologic processes, pathogenic processes, or pharmacologic responses to a therapeutic intervention. ${ }^{3}$ A good biomarker should be specific and sensitive and have the ability to inform about the biological condition in terms of periodontal tissue changes and their relationships with the particular phase of OTM.

Table Showing Biomarkers of Orthodontic Tooth Movement:

\begin{tabular}{|l|l|l|}
\hline $\begin{array}{l}\text { Metabolic products of para dental } \\
\text { remodeling }\end{array}$ & Inflammatory mediators & Enzymes and enzyme inhibitors \\
\hline Glycosaminoglycans & Prostaglandin E & Cathepsin B \\
\hline Pyridinium derivatives & $\begin{array}{l}\text { Neuropeptides (Calcitonin related gene peptide } \\
\text { and substance P) }\end{array}$ & $\begin{array}{l}\text { Acid phosphatase and alkaline } \\
\text { phosphatase }\end{array}$ \\
\hline Pantraxin 3 & Transforming growth factora1 & Glucuronidase \\
\hline N-telopeptide type 1 andosteocalcin & Epidermal growth factor & Aspartate aminotransferase \\
\hline Matrix metalloproteins $1 \& 8$ & a2 microglobulin and insulin like growth factor 1 & Lactate dehydrogenase \\
\hline & IL1 receptor antagonist 1a,2,6,8 & \\
\hline & Tumor necrosis factor & \\
\hline & Macrophage-CSF & \\
\hline & RANK/RANKL/osteoprotegerin system & \\
\hline & Myeloperoxidase & Markers of root resorption \\
\hline
\end{tabular}

Metabolic products of para dental remodeling: Glycosaminoglycans (GAG) were investigated in the flow of GCF at three stages of orthodontic treatment viz. before orthodontic treatment, during canine retraction and in retention, to relate them to tooth movement. Studies concluded that the increase in GCF volume during OTM and the decrease during retention were only partly due to changes in the severity of gingival 
inflammation (Pender et al). The pyridinium derivatives, pyridinoline (Pyr) and deoxypyridinoline ( $\mathrm{dPyr})$, are structural elements that bind together collagen chains. Pyr is abundant in skeletal tissues, whereas dPyr is a minor component found predominantly in bone and dentin. These two molecules are used as markers to evaluate bone resorption in such cases as Paget's disease and primary hyperparathyroidism. ${ }^{5}$ Pentraxin3 (PTX3), also known as tumor necrosis factor (TNF) stimulated gene 14 (TSG14), is a $45 \mathrm{kDa}$ glycoprotein with a 202 amino acids Surlinet al.[12] measured the levels of PTX3 in GCF in orthodontic young and adult patients in the first 2 weeks after the orthodontic appliance showing an increased GCF levels of PTX3 suggesting PTX3 involvement in periodontal orthodontic remodeling and the aseptic inflammation induced by the orthodontic forces.N-telopeptide (NTx) is a specific marker of bone resorption because of its crosslinked a2 (I) NTx. When multiple biochemical markers of bone turnover were compared, NTx was found to be a more sensitive measure of bone resorption. Hence, NTx might be an important marker of active periodontal bone loss and could be useful for analyzing site specific responses to periodontal therapy. Osteocalcin is a noncollagenous matrix protein of calcifying and calcified tissue. It is produced by osteoblasts and has been described as the most specific marker of osteoblast function. Matrix metalloproteins (MMPs) are chemokines may contribute to differential bone remodeling in response to orthodontic forces through the establishment of distinct microenvironments in the sites of both compression and tension. ${ }^{6}$ MMPs are enzymes that play a central role in PDL remodeling, both in physiological and in pathological conditions.

Inflammatory Mediators: Prostaglandin E (PGE2), specially, is able to mediate inflammatory responses and induce bone resorption by activating osteoclastic cells. $^{7}$ Prostaglandins (PGs) are a group of chemical messengers and are derivatives of arachidonic acid. It has been found that PGs have an important role in promoting bone resorption. Although the exact role of PGs in bone resorption is not clear, it is thought to do so by stimulating cells to produce cyclic adenosine monophosphate, which is an important chemical messenger for bone resorption. Research proved that the application of orthodontic force increased the synthesis of PGs, which in turn stimulated osteoclastic bone resorption. The peripheral sensory nervous system contributes to the development of acute and chronic inflammatory processes through the local release of neuropeptides. ${ }^{[20,21,22]}$ With the application of physiologic orthodontic force, SP increases production of proinflammatory cytokines and formation of osteoclasts in dental pulp fibroblasts in patients with severe orthodontic root resorption. ${ }^{8}$ Transforming growth factor is a family of polypeptides produced by cells within the periodontium involved in many biologic activities, including cell growth, differentiation and apoptosis, as well as in developmental processes and bone remodeling. ${ }^{9}$ Epidermal growth factor (EGF) is another cytokine possibly associated with bone remodeling. Fibroblasts and stromal cells produce it. ${ }^{10}$ Uematsuet al. in a study reported a transient elevation of EGF levels in GCF after application of mechanical stress of an experimental tooth. Alfa- 2 microglobulin ( $\alpha 2 \mathrm{MG})$ enhances the biologic action of insulinlike growth factor I (GF). ${ }^{11}$ They are a family of peptides that promote cell proliferation and differentiation and have insulin like metabolic effects. They have been associated with stimulation of the osteoblasts and its functions. ${ }^{12}$

Interleukin1 (IL1) are cytokines that affect bone metabolism and OTM, has 2 forms - á and â- that code different genes have similar actions. It was recently found that the concentration of leptin in GCF is decreased by orthodontic orthodontic tooth movement and this conclusively proved that leptin may have been one of the mediators responsible for orthodontic tooth movement. IL-17 has been found to be increased in patients with periodontitis, while it was barely detectable in sera from periodontally healthy individuals. Tumor necrosis factor-a, another proinflammatory cytokine, was shown to elicit acute or chronic inflammation and stimulate bone resorption. TNF-a is a pro-inflammatory cytokine that is often overexpressed in periodontitis and is responsible for alveolar bone resorption during periodontal breakdown. TNF-a plays a pivotal role in the bone resorption process, thus helping in orthodontic tooth movement.Colony stimulating factors are specific glycoproteins, which interact to regulate production, maturation and function of monocyte macrophages CSF (MCSF) as well as granulocytes CSF (GCSF). They might have implications in bone remodeling and thereby during tooth movement. ${ }^{13}$ An important implication in tooth movement is played by the MCSF through an increased early osteoclastic recruitment and differentiation. ${ }^{14}$ In the future, optimal dosages of MCSF already correlated with measurable changes in tooth movement and gene expression will provide a great potential in accelerating clinically the rate of tooth movement. The TNF related 
ligand receptor activator of nuclear factor kappa ligand (RANKL) and its two receptors, receptor activator of nuclear factor kappa (RANK) and osteoprotegerin (OPG), are known for involvement in bone remodeling process. In the bone system, RANKL is expressed on osteoblast cell lineage and exerts its effect by binding the RANK receptor on osteoclast lineage cells. This binding leads to rapid differentiation of hematopoietic osteoclast precursors to mature osteoclasts. Osteoprotegerin is a decoy receptor produced by osteoblastic cells, which compete with RANK for RANKL binding. The biologic effects of OPG on bone cells include inhibition of terminal stages of osteoclast differentiation, suppression of the activation of matrix osteoclasts and induction of apoptosis. Myeloperoxidase (MPO) is an enzyme found in polymorphonuclear neutrophil (PMN) granules and can be used to estimate the number of PMN granules in the tissues. Mean MPO activity increased in both the GCF and saliva of orthodontic patients $2 \mathrm{~h}$ after appliance activation and they might be a good biomarker to assess inflammation in orthodontic movement.

Enzymes and enzyme inhibitors: Cathepsin B, an intracellular lysosomal enzyme is known to play an important role in the initiation and perpetuation of inflammatory processes. The accumulation of cathepsin $\mathrm{B}$ in GCF has been shown to increase with OTM. They were increased around osteoclasts and played a role in the decomposition of exposed collagen fibers and collagen degradation byproducts. ${ }^{15,16}$ Alkaline phosphatase and acid phosphatase have been examined as bone turn over markers in orthodontic tooth movement. ${ }^{17}$ Bone metabolismis associated with alkaline phosphatase (ALP) and acid phosphatase (ACP), expressed, respectively, by osteoblasts and osteoclasts. Alkaline phosphatase is a ubiquitous tetrameric enzyme, localized outside the cell membrane. ${ }^{18} \mathrm{~A}$ biomarker of primary granule release from polymorphonuclear leukocytes is the lysosomal enzyme $\beta$ glucuronidase ( $\beta \mathrm{G}$ ). Increased levels of this enzyme have been found in the GCF of adolescents treated with the rapid maxillary expander. Moreover, $\beta$ $\mathrm{G}$, as other biochemical mediators like IL1 $\beta$, responds to direct and indirect application of mechanical force to teeth, with an increased level that is higher than following stronger forces. ${ }^{19,20}$ Aspartate aminotransferase (AST) is a soluble enzyme that is normally confined to the cytoplasm of cells, but is released to the extra-cellular environment upon cell death. The activity levels of AST in the GCF are considered to be important in regulating alveolar bone resorption during orthodontic tooth movement. Aspartate aminotransferase (AST) and lactate dehydrogenase activities in GCF have been measured to confirm the biological activity, which occurs in the periodontium during orthodontic treatment. They are soluble enzymes normally confined to the cytoplasm of cells then released to the extracellular environment after cell necrosis. ${ }^{21}$ Lactate dehydrogenase, an enzyme normally limited to the cytoplasm of cells, signals an increase in LDH during orthodontic tooth movement due to changes in the periodontal ligament. ${ }^{22}$ Lactate dehydrogenase (LDH), an enzyme normally limited to the cytoplasm of cells, is only released extra cellularly after cell death.

\section{Conclusion}

The orthodontic displacement of a tooth is the result of a mechanical stimulus, generated by a force applied to the crown of a tooth, which results in an acute inflammatory response in periodontal tissues, which in turn may trigger the cascade of biological events associated with bone remodeling. Orthodontic force application could be based on individual tissue responses. The problem of relapse can be solved upto some extent by considering bone turnover rates around each experimental tooth, however a simple noninvasive method is required for achieving these possibilities. The gingival crevicular fluid alkaline phosphatase levels in can be used as a diagnostic biomarker to assess the health and pathology of the periodontium during orthodontic treatment. It can be used in early detection of changes in the periodontium and can assess the efficacy and prognosis of orthodontic treatment.

Ethical Clearance: Not needed

\section{Source of Funding: self}

\section{Conflict of Interest: Nil}

\section{References}

1. Krishnan V, DavidovitchZ. Cellular, molecular and tissue level reactions to orthodontic force. Am J Orthod Dentofacial Orthop. 2006;129:469.

2. Dolce C, Malone JS, Wheeler TT. Current concepts in the biology of orthodontic tooth movement. SeminOrthod. 2002;8:6-12.

3. BartzelaT, Türp JC, Motschall E, Maltha JC. Medication effects on the rate of orthodontic tooth movement: A systematic literature review. Am J Orthod Dentofacial Orthop. 2009;135:16-26. 
4. Burstone CJ. The biomechanics of tooth movement. In: Kraus BS, Riedel RA, editors. Vistas in Orthodontics. Philadelphia: Lea and Febiger; 1962.

5. Pilon JJ, Kuijpers Jagtman AM, Maltha JC. Magnitude of orthodontic forces and rate of bodily tooth movement. An experimental study. Am J Orthod Dentofacial Orthop. 1996;110:16-23.

6. Van Leeuwen EJ, Maltha JC, KuijpersJagtman AM. Tooth movement with light continuous and discontinuous forces in beagle dogs. Eur J Oral Sci. 1999;107:468-74.

7. Taba M, Jr, Kinney J, Kim AS, Giannobile WV. Diagnostic biomarkers for oral and periodontal diseases. Dent Clin North Am. 2005;49:551-71.

8. Last KS, Donkin C, Embery G. Glycosaminoglycans in human gingival crevicularfluid during orthodontic movement. Arch Oral Biol. 1988;33:907-12.

9. Samuels RH, Pender N, Last KS. The effects of orthodontic tooth movement on the glycosaminoglycan components of gingival crevicular fluid. J Clin Periodontol.1993;20:371-77.

10. Pender N, Samuels RH, Last KS. The monitoring of orthodontic tooth movement over a 2 year period by analysis of gingival crevicular fluid. Eur J Orthod. 1994;16:511-20.

11. Griffiths GS, Moulson AM, Petrie A, James IT. Evaluation of osteocalcin and pyridinium crosslinks of bone collagen as markers of bone turnover in gingival crevicular fluid during different stages of orthodontic treatment. J Clin Periodontol. 1998;25:492-8.

12. Surlin P, Rauten AM, Silosi I, Foia L. Pentraxin3 levels in gingival crevicular fluid during orthodontic tooth movement in young and adult patients. Angle Orthod. 2012;82:833-8.

13. Alfaqeeh SA, Anil S. Osteocalcin and Ntelopeptides of type I collagen marker levels in gingival crevicular fluid during different stages of orthodontic tooth movement. Am J Orthod Dentofacial Orthop. 2011;139:e553-9.

14. Garlet TP, Coelho U, Repeke CE, Silva JS,
Cunha Fde Q, Garlet GP. Differential expression of osteoblast and osteoclast chemmoatractants in compression and tension sides during orthodontic movement. Cytokine. 2008;42:330-5.

15. Cantarella G, Cantarella R, Caltabiano M, Risuglia $\mathrm{N}$, Bernardini R, Leonardi R. Levels of matrix metalloproteinases 1 and 2 in human gingival crevicular fluid during initial tooth movement. Am J Orthod Dentofacial Orthop. 2006;130:568.e11-6.

16. Grieve WG, 3rd, Johnson GK, Moore RN, Reinhardt RA, DuBois LM. Prostaglandin E (PGE) and interleukin1 beta (IL1 beta) levels in gingival crevicular fluid during human orthodontic tooth movement.Am J Orthod Dentofacial Orthop. 1994;105:369-74.

17. Dudic A, Kiliaridis S, Mombelli A, Giannopoulou C. Composition changes in gingival crevicular fluid during orthodontic tooth movement: Comparisons between tension and compression sides. Eur J Oral Sci. 2006; 114:416-22.

18. Groeneveld MC, Everts V, Beertsen W. Alkaline phosphatase activity in the periodontal ligament and gingiva of the rat molar: Its relation to cementum formation. J Dent Res. 1995;74:1374-81

19. Klein DC, Raisz LG. Prostaglandins: Stimulation of bone resorption in tissue culture. Endocrinology. 1970;86:1436-40.

20. Olgart L, Gazelius B. Effects of adrenaline and felypressin (octapressin) on blood flow and sensory nerve activity in the tooth. Acta Odontol Scand. 1977;35:69-75.

21. Perinetti G, Paolantonio M, D'Attilio M, D'Archivio D, Dolci M, Femminella B, et al. Aspartate aminotransferase activity in gingival crevicular fluid during orthodontic treatment. A controlled short term longitudinal study. J Periodontol. 2003;74:145-52.

22. Luthman J, Dahllöf G, Modèer T, Johansson O. Immunohistochemical study of neuronal markers in human gingiva with phenytoininduced overgrowth. Scand J Dent Res. 1988;96:339-46. 